\title{
Differences in Zbtb7a expression cause heterogeneous changes in human nasopharyngeal carcinoma $\mathrm{CNE} 3$ sublines
}

\author{
FEI LIU ${ }^{1}$, JIAO LAN ${ }^{1}$, WEI JIAO ${ }^{1}$, XIANGLAN MO $^{2}$, YONGTA HUANG $^{2}$, HUILAN YE $^{3}$, \\ RUIPING XIAO ${ }^{1}$, YONGLI WANG ${ }^{4}$, MINGZHENG MO ${ }^{1}$ and LIWEI SHI ${ }^{5}$ \\ ${ }^{1}$ Research Center of Medical Sciences, Departments of ${ }^{2}$ Pathology, ${ }^{3}$ Gastroenterology and ${ }^{4}$ Otolaryngology-Head \\ and Neck Oncology, The People's Hospital of Guangxi Zhuang Autonomous Region, Nanning, Guangxi 530021; \\ ${ }^{5}$ Vaccine Clinical Research Institute, Guangxi Center for Disease Prevention \\ and Control, Nanning, Guangxi 530028, P.R. China
}

Received September 27, 2015; Accepted April 6, 2017

DOI: $10.3892 / \mathrm{ol} .2017 .6553$

\begin{abstract}
The present study aimed to determine the association between changes in Zbtb7a expression levels and heterogeneity of nasopharyngeal carcinoma (NPC) CNE3 sublines. CNE3 sublines were established by screening of serial dilution and continuous passage. Proliferative ability and tumorigenicity of the sublines were analyzed separately by soft-agar colony formation and mouse studies. The NPC tissues from mice were analyzed by histological evaluation and immunohistochemistry. The expression levels of Zbtb7a mRNA and protein were analyzed separately by quantitative reverse transcription polymerase chain reaction and western blotting. According to findings from the soft-agar colony formation and mouse studies, two sublines with increased tumorigenicity compared with other sublines were transfected transiently with Zbtb7a short hairpin RNA (shRNA) recombinant plasmid. The changes in viability, migration and invasion abilities were evaluated separately by MTT, colorimetric focus-formation, Transwell migration and invasion assays. The sublines CNE3-GX6 and CNE3-GX11 were selected for subsequent study due to increased tumorigenicity and increased Zbtb7a expression levels compared with the other sublines. High metastatic potency was not observed in all of the sublines. Zbtb7a expression levels were positively associated with tumorigenic degree of the sublines. The growth, migration and invasion abilities of the sublines transfected
\end{abstract}

Correspondence to: Dr Wei Jiao, Research Center of Medical Sciences, The People's Hospital of Guangxi Zhuang Autonomous Region, 6 Taoyuan Road, Nanning, Guangxi 530021, P.R. China E-mail: gxjw2005@gmail.com

Abbreviations: NPC, nasopharyngeal carcinoma; NAC, nasopharyngeal adenocarcinoma; IHC, immunohistochemistry; $\mathrm{H} \& \mathrm{E}$, hematoxylin and eosin; shRNA, short hairpin RNA; BC, blank control; NC, negative control; ln, lymph node

Key words: nasopharyngeal carcinoma, Zbtb7a, CNE3, sublines, heterogeneity with Zbtb7a shRNA plasmid were decreased compared with the cells transfected with empty vector in the negative control group. The findings suggest Zbtb7a expression levels may be associated with heterogeneity of CNE3 sublines. Therefore, Zbtb7a may have an important role in the regulatory mechanism of NPC heterogeneity.

\section{Introduction}

It is well known that recurrence and metastasis are important causes of mortality in nasopharyngeal carcinoma (NPC) patients (1). Unfortunately, the mechanisms underlying the metastasis of NPC remain unclear. Therefore, there is an urgent need to investigate the mechanisms. The CNE3 cell line was obtained from liver metastatic carcinoma of primary NPC and established in 1992 (2). A molecular pathological study has previously indicated that the histological type of the CNE3 cell line transformed from an undifferentiated non-keratinizing carcinoma with focal adenocarcinoma differentiation into a poorly differentiated adenocarcinoma (3). The results provided approaches for studying metastatic NPC.

Tumor heterogeneity is a subclonal process, where clones of cancer cells may differ in characteristics, including karyotype, invasiveness, growth rate, expression of cell surface markers and sensitivity to therapeutics $(4,5)$. According to Paget's theory, metastasis is clonal in its nature (6). Therefore, heterogeneous characteristics of clonal sublines are an important reason that malignant tumor is inclined to recurrence and metastasis in the advanced stages (7). Successful construction of tumor sublines will provide an efficient tool for screening metastasis-associated genes and investigating invasive and metastatic mechanisms $(8,9)$.

The pro-oncogene Zbtb7a is also named Pokemon/ FBI-1/OCZF/LRF, containing broad complex, tramtrack, bric-a-brac/poxvirus and zinc finger (BTB/POZ) domain and actin-binding repeats $(10,11)$. Zbtb7a has a critical role in oncogenesis (12). Zbtb7a is able to repress transcription of $\mathrm{Rb}$ via its POZ domains in different cancer cell lines (13), activate transcription in fatty-acid synthase promoter and provide more phospholipid membrane components required for rapid proliferation of cancer cells (14). It has been reported that Zbtb7a 
is closely associated with a number of different cancer types, including breast cancer (15), prostate cancer (16), hepatocellular carcinoma (17), NPC (18) and colorectal cancer (19). Early studies by the present authors demonstrated that increasing expression levels of Zbtb7a mRNA and protein were accompanied by NPC progression in most cases (18). Subsequently, short hairpin RNA (shRNA)2 plasmids were constructed and screened for their ability to knock down Zbtb7a expression (20). In the present study in order to elucidate the characteristics of the CNE3 cell line from NPC distant metastases, a number of sublines were established by single cell cloning and stable passaging. Sublines with different tumorigenicity were subsequently selected and heterogeneity in cellular characteristics was investigated. Finally, the associations between changes in Zbtb7a expression and heterogeneity in cellular characteristics were analyzed.

\section{Materials and methods}

Cell culture. The human NPC epithelial cell line CNE3 was originally obtained and preserved at the Research Center of Medical Sciences, The People's Hospital of Guangxi Zhuang Autonomous Region in Nanning (Guangxi, China) (2). The CNE3 cells were grown in RPMI-1640 medium with $10 \%$ fetal bovine serum (FBS; Gibco; Thermo Fisher Scientific, Inc., Waltham, MA, USA).

Single cell cloning by serial dilution. The single CNE3 cell clones were screened by serial dilution. A total of $100 \mu \mathrm{l}$ complete culture medium was added to 95 wells in the 96-well plate (Corning Incorporated, Corning, NY, USA). A total of $200 \mu \mathrm{l}$ CNE3 cell suspension $\left(5 \times 10^{4} / \mathrm{ml}\right)$ was added to one well (A1), and $100 \mu 1$ suspension was transferred from well A1 to B1 and mixed by gentle pipetting. The 1:2 dilutions were repeated down the entire column, and $100 \mu 1$ suspension was discarded from well H1. A total of $100 \mu \mathrm{l}$ additional medium was added to each well in the first column, and $100 \mu 1$ suspension was transferred from the first column to the second column. The 1:2 dilutions were repeated across the entire plate, and $100 \mu \mathrm{l}$ medium was added to each well.

Soft agar colony formation. Thirteen CNE3 sublines were established by screening single cell clones and stable passaging. The sublines were named sequentially from CNE3-GX1 to CNE3-GX13. A base layer comprising $2 \mathrm{ml}$ medium $(0.5 \%$ agarose; Beijing Solarbio Science \& Technology Co., Ltd., Beijing, China) in a 6-well plate (Corning Incorporated) was solidified at $4^{\circ} \mathrm{C}$ for $15 \mathrm{~min}$. A cell layer containing $10^{3}$ cells in $1 \mathrm{ml}$ medium $\left(0.25 \%\right.$ agarose) was solidified at $4^{\circ} \mathrm{C}$ for $15 \mathrm{~min}$ and subsequently incubated at $37^{\circ} \mathrm{C}$ under $5 \% \mathrm{CO}_{2}$. The number of colonies (over 50 cells/colony) was counted after 14 days of incubation using a CKX41 microscope (original magnification, $\mathrm{x} 40$; Olympus Corporation, Tokyo, Japan).

Xenograft mouse tumor model. Based on the results of soft agar colony formation and cell morphology, 5 sublines and CNE3 were selected to establish tumor model. A total of $60 \mathrm{BALB} / \mathrm{c}$ mice were obtained from the Guangxi Medical University Laboratory Animal Centre. The mice were female, 4-weeks old and weighed $\sim 15 \mathrm{~g}$, and were randomly divided into six groups. Mice were raised in independent ventilation cages (Tecniplast S.p.A, Buguggiate, Italy). The temperature was $20-25^{\circ} \mathrm{C}$. The atmosphere was $20-50 \mathrm{~Pa}$. The light/dark cycle was 10/14 h. The food and water were sterilized. A nude mouse was subcutaneously injected with cells $\left[10^{7} / 200 \mu 1\right.$ phosphate-buffered saline (PBS)] from different sublines. The end point of when the mice were sacrificed was 8 weeks. Then they were anatomized and evaluated for the probability of metastasis after 8 weeks. The weight and volume of primary tumor were measured. The protocol for establishing the transplanted tumor model of CNE3 was the same as the tumor model of CNE3 sublines. Tumor volume was calculated by using the formula: $\mathrm{V}=(\pi / 6)(\mathrm{d} 1 \mathrm{xd} 2)^{3 / 2}$. The present study was approved by the Ethics Committee of the People's Hospital of Guangxi Zhuang Autonomous Region (Nanning, China).

Histological evaluation and immunohistochemistry (IHC). Histological evaluation and IHC were performed as previously described (3). The tissues were obtained from nude mice transplanted with tumors of cells from the CNE3 cell line and its sublines. The tissues were fixed for $24 \mathrm{~h}$ by $10 \%$ neutral formalin, at room temperature. Tissues were then sliced into $4-\mu \mathrm{m}$ thick sections. Tissues were stained with hematoxylin and eosin (H\&E); $0.5 \%$ hematoxylin stained for $5 \mathrm{~min}$ and $0.5 \%$ eosin stained for $2 \mathrm{~min}$ at room temperature. A non-biotin horseradish peroxidase ready-to-use two-step detection system (ZSGB-BIO, Beijing, China) was used in the IHC analysis. There was an increased abundance of positive staining observed in the brown granules compared with the unspecific background staining. Staining was primarily observed in the cell nucleus (p63) or cytoplasm [cytokeratin (CK)5/6, CK7]. The positive cell rates and staining intensities were analyzed in the intact slices by high power fields (original magnification, $\mathrm{x} 200$ ). The results of the positive cell rates $(<10 \%)$ and weak coloring were negative. The results of the positive cell rates $(>10 \%)$ and dark brown granules were positive. Staining was determined as positive when $>10 \%$ of the cells were positively stained, and positive staining was observed in the dark brown granules. H\&E and IHC were analyzed using a BX51 microscope (Olympus Corporation).

Quantitative reverse transcription polymerase chain reaction (RT-qPCR) and western blotting. RT-qPCR and western blot analysis were performed as previously described (18). For RT-qPCR, total RNA from the CNE3-GX4, CNE3-GX6, CNE3-GX7, CNE3-GX10 and CNE3-GX11 sublines was extracted by TRIzol (Invitrogen; Thermo Fisher Scientific, Inc.). RNA amount was detected by NanoDrop 2000 (Thermo Fisher Scientific, Inc.). Total RNA (4 $\mu \mathrm{l})$ was analyzed by $1.2 \%$ agarose gel electrophoresis with $0.6 \mathrm{~mol} / \mathrm{l}$ formaldehyde. The gel was photographed by Bio Imaging System Gene Genius (Syngene, Cambridge, UK). An equal amount $(4 \mu \mathrm{g})$ of total RNA was synthesized as a first-strand cDNA using the RevertAid ${ }^{\mathrm{TM}}$ First-Strand cDNA Synthesis kit (Fermentas; Thermo Fisher Scientific, Inc.) according to the manufacturer's protocol. The cDNA was the source of the template. The Zbtb7a (NM_015898.2) primers were: Forward, 5'-GCTTGGGCC GGTTGAATGTA-3' and reverse, 5'-GGCTGTGAAGTTACC GTCGG-3'. GAPDH was used as an internal reference control. The GAPDH (NM_002046.4) primers: forward, 5'-CATGAG 
AAGTATGACAACAGCC-3' and reverse, 5'-AGTCCTTCC ACGATACCAAAGT-3'. All primer sequences were designed and synthesized by Invitrogen (Thermo Fisher Scientific, Inc.). The reaction mixture consisted of $10 \mu \mathrm{l}$ Super Real PreMix and $0.4 \mu \mathrm{l}$ 50X ROX (both from Tiangen Biotech Co., Ltd., Beijing, China), $0.5 \mu \mathrm{l}$ template, $0.5 \mu \mathrm{l}$ forward primer, $0.5 \mu \mathrm{l}$

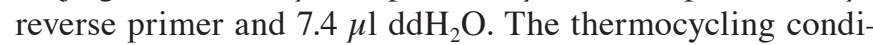
tions included an initial denaturation step at $95^{\circ} \mathrm{C}$ for $15 \mathrm{~min}$, denaturation at $95^{\circ} \mathrm{C}$ for $10 \mathrm{sec}$ and annealing at $60^{\circ} \mathrm{C}$ for $32 \mathrm{sec}$ for 40 cycles, dissociation stage at $95^{\circ} \mathrm{C}$ for $15 \mathrm{sec}$, $60^{\circ} \mathrm{C}$ for $1 \mathrm{~min}, 95^{\circ} \mathrm{C}$ for $15 \mathrm{sec}, 60^{\circ} \mathrm{C}$ for $15 \mathrm{sec}$. The reaction program was executed by the 7500 Real-Time PCR system (Applied Biosystems; Thermo Fisher Scientific, Inc.). The expression level of Zbtb7a mRNA was calculated using the $\Delta \Delta$ Cq method (21).

For western blotting, protein $(20 \mu \mathrm{g})$ was subjected to $10 \%$ SDS-PAGE and transferred onto $0.22 \mu \mathrm{m}$ PVDF membranes (Millipore, Billerica, MA, USA) using the Mini-Protean system (Bio-Rad Laboratories, Inc., Hercules, CA, USA). The membranes were incubated with the primary antibodies against Zbtb7a (1:500 dilution; catalog no. ab70208; Abcam, Cambridge, UK) and $\beta$-actin (1:1,200 dilution; catalog no. AA128; Beyotime Institute of Biotechnology, Shanghai, China) overnight at $4^{\circ} \mathrm{C}$. The membranes were subsequently incubated with peroxidase-conjugated goat anti-rabbit IgG (H+L; 1:8,000 dilution; catalog no. ZB-2301; ZSGB-BIO) or horseradish peroxidase-labeled goat anti-mouse $\operatorname{IgG}(\mathrm{H}+\mathrm{L}$; 1:12,000 dilution; catalog no. A0216; Beyotime Institute of Biotechnology) for $2 \mathrm{~h}$ at $37^{\circ} \mathrm{C}$. The detection was performed using the BeyoECL Plus kit (Beyotime Institute of Biotechnology) according to the manufacturer's protocol. Fuji Medical X-ray Film (Guangxi Yesstar Medical Systems Co., Ltd., Nanning, Guangxi, China) was placed on top of the membrane and performed exposure. The exposed X-ray film was scanned (Unisplendour Co., Ltd., Beijing, China).

Transient knockdown of Zbtb7a in CNE3 sublines. The sublines CNE3-GX6 and CNE3-GX11, with increased tumorigenicity compared with other sublines, as demonstrated by soft agar colony formation assay, animal experiments and analysis of Zbtb7a expression levels, were selected. The pRNAT-U6.1/Neo (GenScript, Piscataway, NJ, USA) vector was used for all transfection experiments, and these were performed as previously described (13). The cells were plated in 6-well plates at a density of $10^{5}$ cells/well, and transient transfection was performed with $4 \mu \mathrm{g}$ plasmid and $10 \mu \mathrm{l}$ Lipofectamine $^{\circledast} 3000$ (Invitrogen; Thermo Fisher Scientific, Inc.) when the cells covered $>50 \%$ of the area. A transfection efficiency of 70-80\% was observed by IX71 fluorescence microscopy (Olympus Corporation) after $48 \mathrm{~h}$, and the transfected cells were used for subsequent assays. The untreated cells and cells transfected with the empty vector and shRNA were named as blank control (BC), negative control (NC) and shRNA, respectively.

MTT assay and colorimetric focus forming assay. The role of Zbtb7a in growth control of CNE3 sublines was analyzed by MTT and focus forming assays. For MTT assay, the cells were plated in 96-well plates at a density of 4,000 cells/well and incubated with 0.5\% MTT (Sigma-Aldrich; Merck KGaA,
Darmstadt, Germany) for $4 \mathrm{~h}$ at $37^{\circ} \mathrm{C}$. Subsequently, the cells were lysed using DMSO (Sigma-Aldrich; Merck KGaA), and the absorbance was detected by using the GF-M3000 ELISA analyzer (Shandong Gaomi Caihong Analytical Instruments Co., Ltd., Gaomi, Shandong, China) at $490 \mathrm{~nm}$. For colorimetric focus forming assay, the NC and shRNA-transfected cells were plated in 6 -well plates at a density of 300 cells/well. Then the cells were fixed using $100 \%$ methanol after 2 weeks of incubation at $37^{\circ} \mathrm{C}$ for $30 \mathrm{~min}$. They were subsequently stained by $5 \%$ crystal violet (Amresco, LLC, Solon, OH, USA) in $100 \%$ methanol at $37^{\circ} \mathrm{C}$ for $10 \mathrm{~min}$. The number of foci (over 50 cells/foci) were counted using a CKX41 microscope (original magnification, $\mathrm{x} 40$; Olympus Corporation).

Transwell migration and invasion assay. Transwell migration and invasion assays were performed in $6.5 \mathrm{~mm}$ Transwell chambers of 24-well plates (pore size, $8 \mu \mathrm{m}$; Costar; Corning Incorporated). A total of $1 \times 10^{5}$ cells (migration assay) and $4 \times 10^{5}$ cells (invasion assay) from the BC, NC and shRNA-transfected groups were resuspended in $200 \mu 1$ serum-free RPMI-1640 medium in the upper chamber. A total of $800 \mu \mathrm{l}$ medium containing $10 \%$ FBS was added to the lower chamber. For invasion assays, the upper surface was coated with $70 \mu \mathrm{l}$ serum-free RPMI-1640 medium (dilution, 1:3) and Matrigel (BD Biosciences, San Jose, CA, USA) prior to the seeding of the cells. The cells were incubated for $4 \mathrm{~h}$ at $37^{\circ} \mathrm{C}$ and $5 \%$ $\mathrm{CO}_{2}$. After $24 \mathrm{~h}$ (migration assay) and $48 \mathrm{~h}$ (invasion assay) of incubation at $37^{\circ} \mathrm{C}$, non-migratory and non-invasive cells in the upper chamber were completely removed by cotton swabs. The cells adhered to the lower chamber were rinsed with PBS, fixed with $100 \%$ methanol and stained with $1 \%$ crystal violet at $37^{\circ} \mathrm{C}$ for $20 \mathrm{~min}$. The number of cells migrated and invaded was quantified by counting using a BX51 microscope (original magnification, $\mathrm{x} 200$ ). For each well, the mean of five individual fields in the center of the filter was obtained.

Statistical analysis. All assays were performed in three independent experiments, and all data are expressed as the mean \pm standard deviation (SD). Data analysis and graphs were performed using Sigma Plot software (version 12.5; SPSS Inc., Chicago, IL, USA). Statistical differences between two groups were evaluated with independent samples t-test, and one-way analysis of variance was used for multiple comparisons. Least significance difference (LSD) test and Student-Newman-Keuls (SNK) test were used as post hoc tests (version 13.0; SPSS Inc.). $\mathrm{P}<0.05$ was considered to indicate a statistically significant difference.

\section{Results}

Soft agar colony forming and morphology of CNE3 sublines. The number of colonies formed was markedly different between the sublines. CNE3-GX10 cells were not able to form any colonies. By contrast, CNE3-GX11 cells formed a significantly increased number of colonies compared with cells from the other sublines (Fig. 1A). These findings suggested that proliferative capability of CNE3-GX10 cells was the weakest and the proliferative capability of CNE3-GX11 cells was the strongest. A total of 5 sublines (CNE3-GX4, CNE3-GX6, CNE3-GX7, CNE3-GX10 and CNE3-GX11) were selected for 

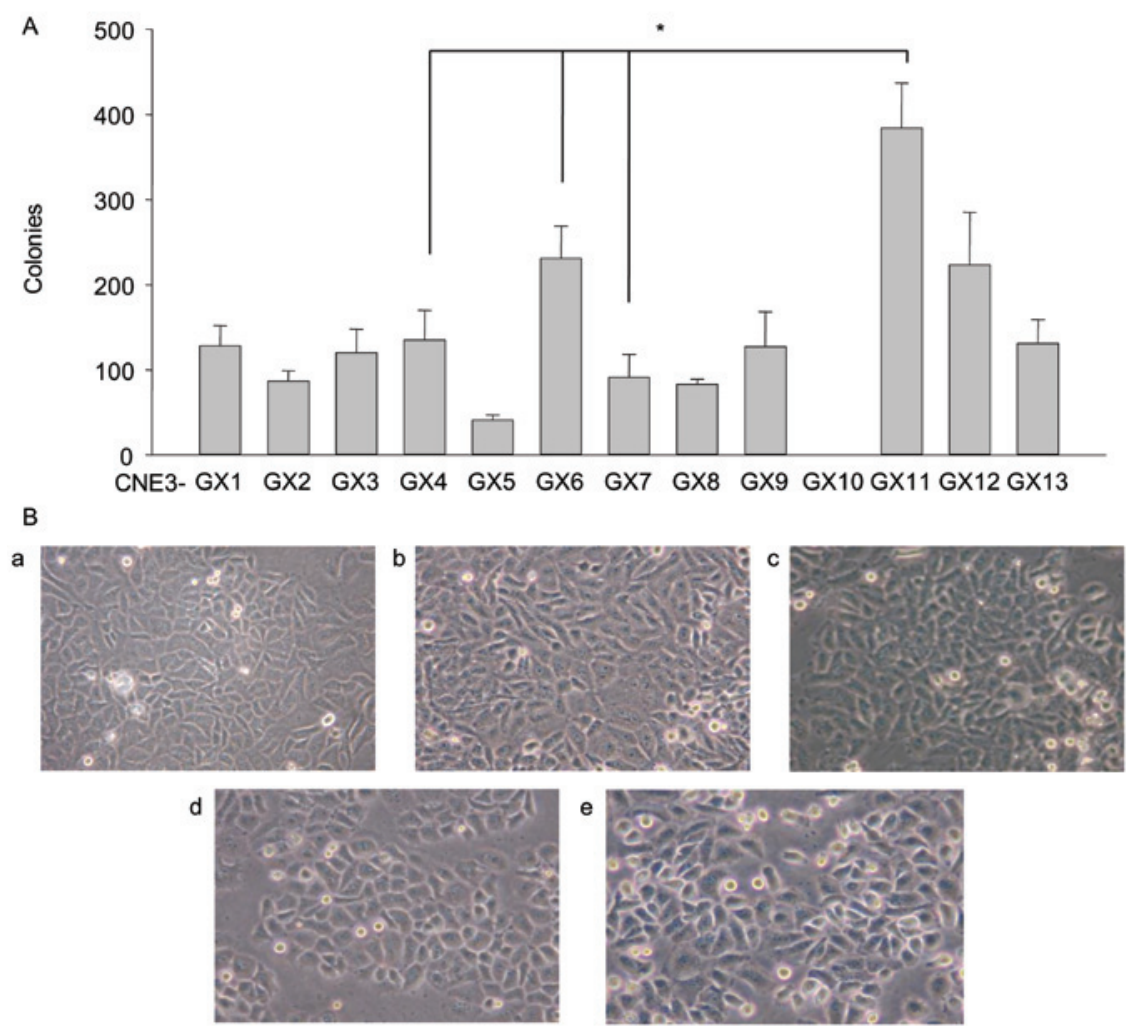

Figure 1. Comparisons of CNE3 sublines in soft agar colony formation and cell morphology. (A) The number of colonies formed in 13 CNE3 sublines. (B) The morphology of 5 CNE3 sublines (magnification, x200): (a) CNE3-GX4, (b) CNE3-GX6, (c) CNE3-GX7, (d) CNE3-GX10 and (e) CNE3-GX11. "P<0.05. In soft agar colony formation, the results of multiple comparisons showed that there was statistical significance among CNE3-GX4/6/7/11.
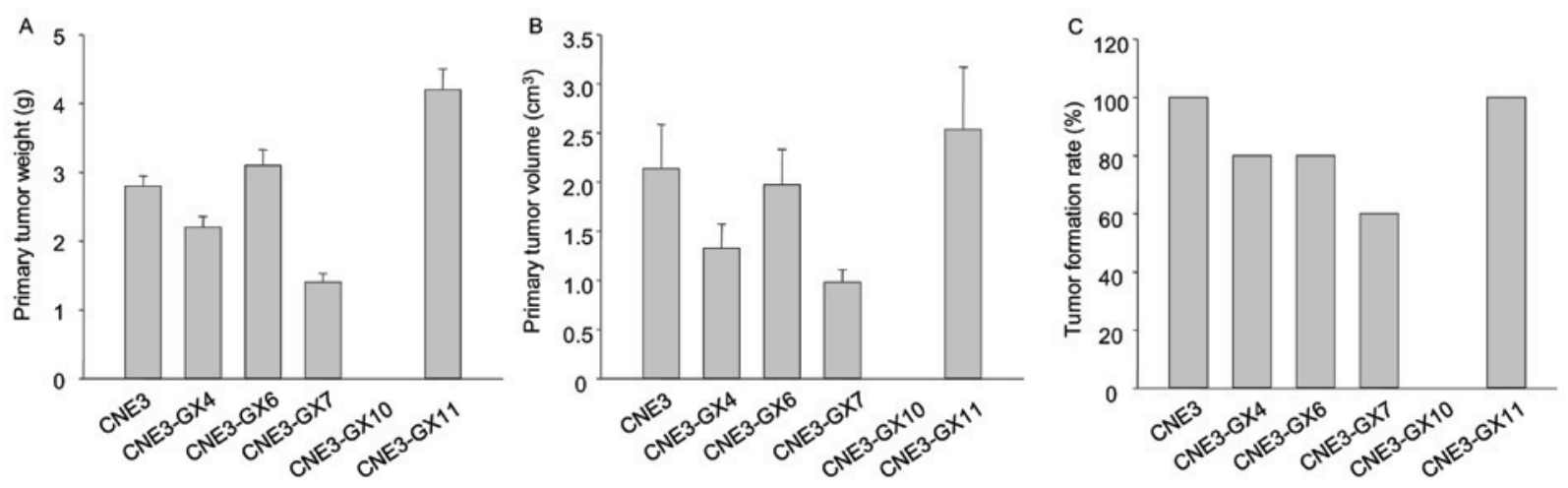

Figure 2. Comparison of tumorigenicity between tumor models established using CNE3 and CNE3-GX4/6/7/10/11. (A) The weight of primary tumor from the CNE3-GX11 tumor model was increased compared with tumor models established using CNE3 and other sublines. (B) The volume of primary tumor of CNE3-GX11 was increased compared with tumors from models established using CNE3 and other sublines. (C) Tumor formation rate of tumor models established using CNE3 and other sublines.

the following experiments according to the obvious differences of soft-agar colony formation and cell morphology (Fig. 1B).

Xenograft mouse tumor model and molecular pathological assays. High metastatic potency was not observed in the sublines CNE3-GX4, CNE3-GX6, CNE3-GX7, CNE3-GX10 and CNE3-GX11. All BALB/c mice injected with CNE3-GX11 cells formed tumors (10/10), while all mice injected CNE3-GX10 cells failed to form tumors $(0 / 10)$. The other sublines (CNE3-GX4, CNE3-GX6 and CNE3-GX7) partly formed tumors. The weight of tumor and volume of tumors formed from CNE3-GX11 cells was increased compared with other sublines (Fig. 2). Findings from histological examination and IHC analysis of tumor tissues formed from CNE3-GX4, CNE3-GX6, CNE3-GX7 and CNE3-GX11 were similar with the results of tumor tissues formed from CNE3 cells (3). H\&E evaluation and IHC revealed that CNE3 sublines had some features of poorly-differentiated adenocarcinoma. CK5/6 and p63 expression was negative and CK7 expression was positive in the IHC assay (3). 

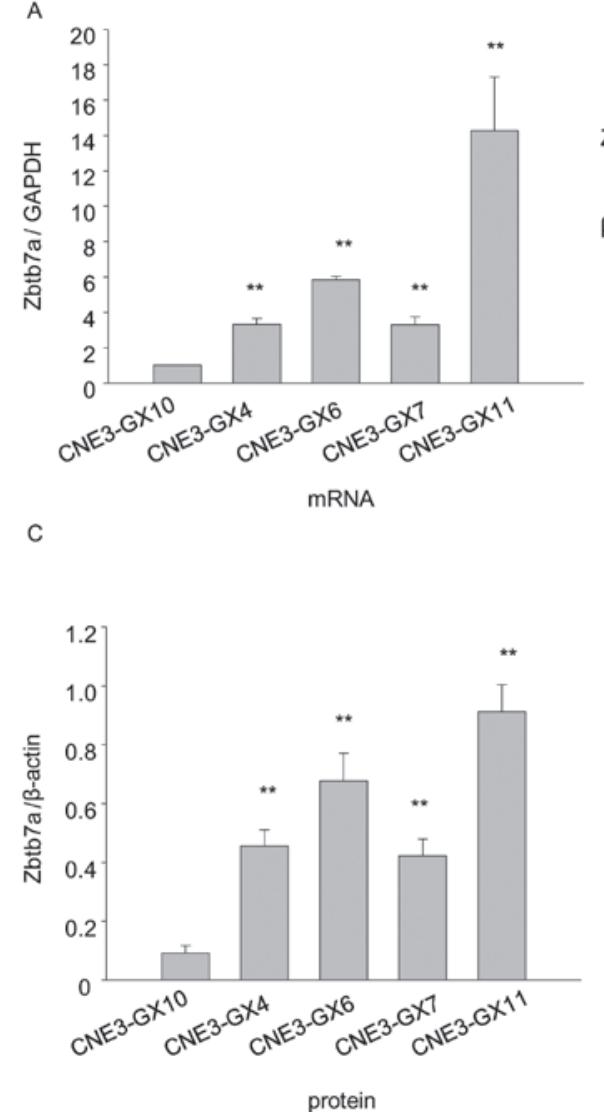

B

Zbtb7a

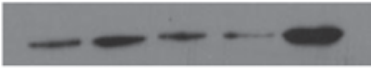

$\beta$-actin

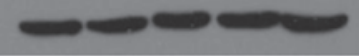

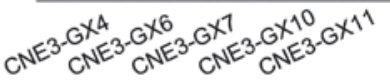

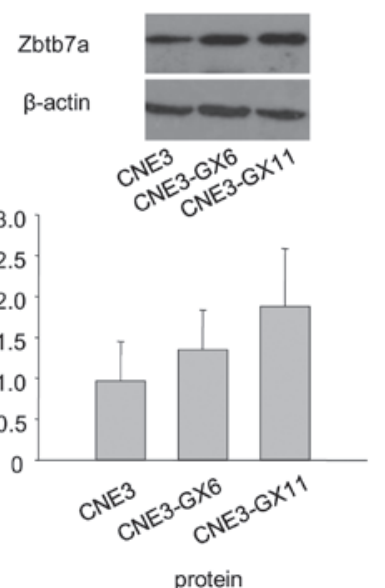

Figure 3. Levels of Zbtb7a expression in CNE3 and CNE3 sublines. (A) The mRNA levels of Zbtb7a in CNE3-GX4/6/7/11 were normalized to the expression in CNE3-GX10. (B) Western blotting was performed to analyze the expression of Zbtb7a proteins. $\beta$-actin was used as an internal control. (C) Densitometric analysis of Zbtb7a expression. (D) The protein levels of Zbtb7a in CNE3-GX6 and CNE3-GX11 were higher than Zbtb7a expression in CNE3 (upper panel). Cumulative results were shown as mean $\pm \mathrm{SD}$ and error bars indicated $\mathrm{SD}$ (lower panel). ${ }^{*} \mathrm{P}<0.05,{ }^{* *} \mathrm{P}<0.01$, compared with $\mathrm{CNE} 3-\mathrm{GX} 10$. SD, standard deviation.

Zbtb7a expression levels of CNE3 and CNE3 sublines. The results indicated that Zbtb7a expression in CNE3-GX11 cells was the highest, while the expression in the CNE3-GX10 cells was the lowest (Fig. 3). Due to their stronger tumorigenicity and higher expression compared with other sublines, CNE3-GX6 and CNE3-GX11 were used for the following assay.

Protein and $m R N A$ levels of Zbtb7a are decreased following Zbtb7a knockdown in CNE3-GX6 and CNE3-GX11. The shRNA recombinant plasmids of Zbtb7a and blank plasmid were transiently transfected into CNE3-GX6 and CNE3-GX11 cells. The results indicated that $\mathrm{Zbtb} 7 \mathrm{a}$ expression was efficiently knocked down in transfected CNE3-GX6 and CNE3-GX11 cells (Fig. 4).

Silencing of Zbtb7a reduces growth, migration and invasion in CNE3-GX6/11. MTT assay showed viability of Zbtb7a shRNA-transfected cells were reduced compared with cells transfected with BC and NC (Fig. 5A and B). Focus forming assay indicated that the number of foci of shRNA-transfected cells was reduced compared with cells transfected with NC (Fig. 5C and D). Transwell migration and invasion assays separately indicated that migratory and invasion abilities of shRNA cells were reduced compared with cells transfected with BC and NC-transfected cells (Fig. 5E and F).

\section{Discussion}

Heterogeneity analysis can be effectively used for researching invasive and metastatic mechanisms of cancer. For example, the SUNE-1 subline has been useful tools for studying tumorigenicity and metastatic potential of NPC, since different heterogeneity was confirmed (22-24).

In the present study, it was demonstrated that there are differences in tumorigenicity, viability, migration and invasion between the CNE3 sublines. Notably, the results indicated that proliferative capability of CNE3-GX10 cells was the weakest and the proliferative capability of CNE3-GX11 cells was the strongest. Similarly, Zbtb7a expression levels and tumorigenicity of CNE3-GX10 was the lowest, while that of CNE3-GX11was the highest. The findings suggest that Zbtb7a expression levels may be associated with heterogeneity of CNE3 sublines.

In order to confirm the hypothesis, RNA interference was used to effectively silence the expression of specific genes through microRNA, small interfering RNA (siRNA) and shRNA. When some genes are transiently knocked down by siRNA or shRNA, some cellular characteristics are temporarily changed (25-27). The cell lines knocked down stably by shRNA can provide an ideal model for studying invasive and metastatic mechanisms of NPC (28). In the present 

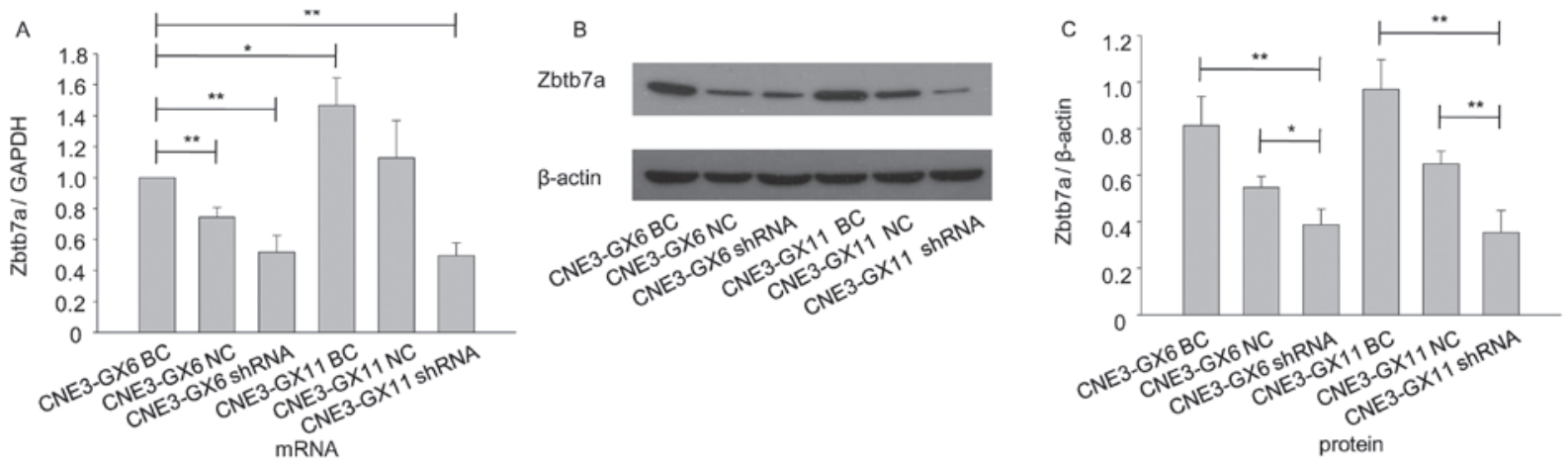

Figure 4. Protein and mRNA levels of Zbtb7a are decreased following transfection in CNE3-GX6/11. (A) The mRNA levels of Zbtb7a in cells transfected with CNE3-GX6 NC/shRNA and CNE3-GX11 BC/NC/shRNA were normalized to the expression in cells transfected with CNE3-GX6 BC. (B) Western blotting was performed to analyze the expression of Zbtb7a proteins. $\beta$-actin was used as internal control. (C) Densitometric analysis of Zbtb7a expression. "P<0.05, ${ }^{* *} \mathrm{P}<0.01$. BC, blank control; NC, negative control; shRNA, short hairpin RNA.

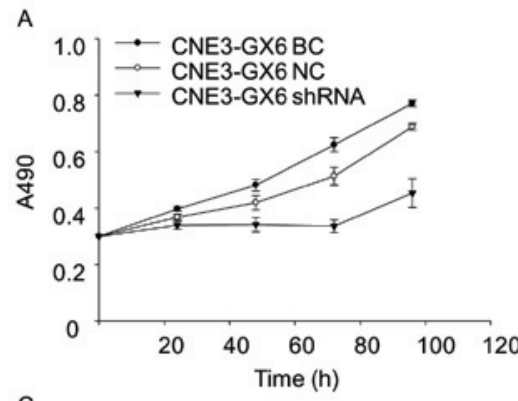

C

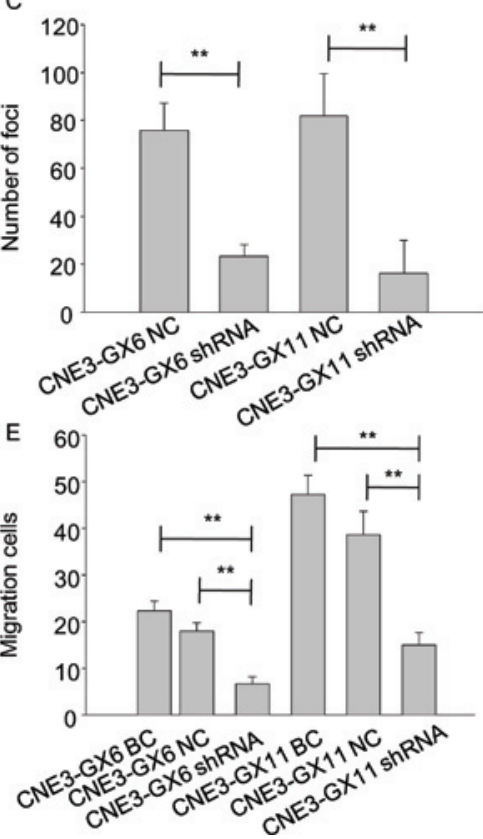

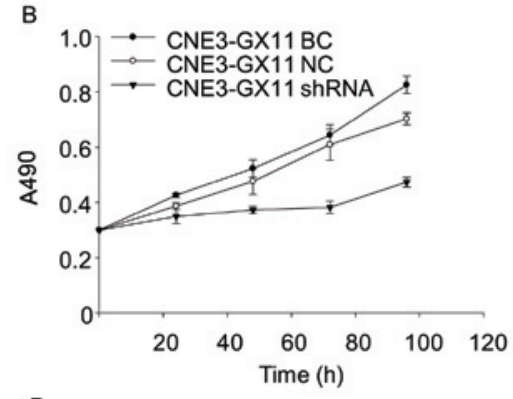
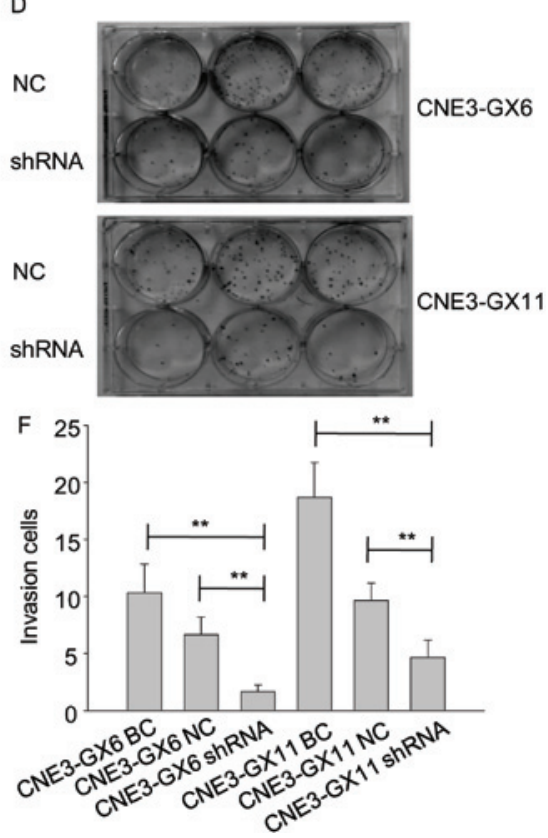

Figure 5. Knockdown of Zbtb7a reduces growth, migration and invasion in CNE3-GX6/11 cell lines. (A) The changes in viability of CNE3-GX6-BC, CNE3-GX6-NC and CNE3-GX6-shRNA transfected cells were evaluated by MTT assay at 0,24,48 and 96h. (B) The changes in viability of CNE3-GX11-BC, CNE3-GX11-NC and CNE3-GX11-shRNA transfected cells were evaluated by MTT assay at 0,24,48 and 96h. (C) The proliferative ability of CNE3-GX6/11-NC and CNE3-GX6/11-shRNA transfected cells was analyzed by colorimetric focus forming assay after 2 weeks of incubation. (D) The foci of the cells were stained by $5 \%$ crystal violate in 6-well plates. (E) The migratory abilities of CNE3-GX6/11-BC, CNE3-GX6/11-NC and CNE3-GX6/11-shRNA-transfected cells were assessed by Transwell assay. (F) The invasion abilities of CNE3-GX6/11-BC, CNE3-GX6/11-NC and CNE3-GX6/11-shRNA-transfected cells were assessed by Transwell assay. ${ }^{* *} \mathrm{P}<0.01$. BC, blank control; NC, negative control; shRNA, short hairpin RNA.

study although high metastatic potency was not observed in the CNE3 sublines, an association between Zbtb7a expression and tumorigenicity was observed. Following transient knockdown of Zbtb7a in CNE3-GX6 and CNE3-GX11 cells, viability, migration and invasion abilities of the cells have decreased. The authors of the present study hypothesize that high Zbtb7a expression may promote tumorigenicity in NPC. 
Why do CNE3 cells lose metastatic ability as a liver metastatic carcinoma cell line? Basing on the 'seed and soil' theory $(5,6,29,30)$, the authors of the present study hypothesize that the tumorigenic characteristics of clonal cells in distant metastasis are altered in order to adapt to new tumor microenvironment for metastasis. Primary nasopharyngeal adenocarcinoma (NAC) is a very rare subtype of NPC (31). CNE3 has typical characteristics of NAC. Since CNE3 was established, it has been used in various studies on NPC (32-37). Therefore, CNE3 is a useful tool for studying characteristics and treatment of NAC.

In summary, the present study suggests that Zbtb7a may have an important impact on the regulatory mechanism of NPC sublines, which may be investigated further. Zbtb7a participates in complex regulation network due to its critical role in cancer. Notably, Zbtb7a acts as a tumor suppressor in colon carcinoma or melanoma cell lines by triggering stable shRNA-mediated knockdown $(38,39)$. It is necessary to investigate whether Zbtb7a acts as a suppressor or not in NPC cell lines with stable knockdown of Zbtb7a. For future studies, the authors of the present study will establish the sublines from high-metastatic potential NPC cell lines such as 5-8F (40) to elucidate the role of Zbtb7a in NPC.

\section{Acknowledgements}

The present study was supported by grants from the Natural Science Foundation of Guangxi Province (grant nos. 2011GXNSFA018308, 2015GXNSFAA139166 and 2016GXNSFBA380144) and the Self Foundation of the Health Department of Guangxi (grant no. Z2013389).

\section{References}

1. Huang PY, Zeng Q, Cao KJ, Guo X, Guo L, Mo HY, Wu PH,Qian CN, Mai HQ and Hong MH: Ten-year outcomes of a randomised trial for locoregionally advanced nasopharyngeal carcinoma: A single-institution experience from an endemic area. Eur J Cancer 51: 1760-1770, 2015.

2. Jiao W: Establishment of a human epithelial cell line of nasopharyngeal carcinoma-CNE3 and its biological characteristics. J Guangxi Med University 12: 187-190, 1995 (In Chinese).

3. Liu F, Jiao W, Mo XL, Lan J, Xiao RP, Zhou XZ, Huang ZL, Mo XM and Li G: Molecular pathological study of the human nasopharyngeal carcinoma CNE3 cell line. Oncol Lett 6: 980-984, 2013.

4. Nowell PC: The clonal evolution of tumor cell populations. Science 194: 23-28, 1976

5. Greaves M and Maley CC: Clonal evolution in cancer. Nature 481: 306-313, 2012.

6. Paget S: The distribution of secondary growths in cancer of the breast. 1989. Cancer Metastasis Rev 8: 98-101, 1889.

7. Bonnet D and Dick JE: Human acute myeloid leukemia is organized as a hierarchy that originates from a primitive hematopoietic cell. Nat Med 3: 730-737, 1997.

8. Li XJ, Ong CK, Cao Y, Xiang YQ, Shao JY, Ooi A, Peng LX, Lu WH, Zhang Z, Petillo D, et al: Serglycin is a theranostic target in nasopharyngeal carcinoma that promotes metastasis. Cancer Res 71: 3162-3172, 2011.

9. Ryszawy D, Sarna M, Rak M, SzPak K, Kedracka-Krok S, Michalik M, Siedlar M, Zuba-Surma E, Burda K, Korohoda W, et al: Functional links between Snail-1 and $\mathrm{Cx} 43$ account for the recruitment of Cx43-positive cells into the invasive front of prostate cancer. Carcinogenesis 35: 1920-1930, 2014.

10. Albagli O, Dhordain P, Deweindt C, Lecocq G and Leprince D: The BTB/POZ domain: A new protein-protein interaction motif common to DNA- and actin-binding Proteins. Cell Growth Differ 6: 1193-1198, 1995.
11. Collins T, Stone JR and Williams AJ: All in the family: The BTB/POZ, KRAB, and SCAN domains. Mol Cell Biol 21: 3609-3615, 2001

12. Maeda T, Hobbs RM, Merghoub T, Guernah I, Zelent A, Cordon-Cardo C, Teruya-Feldstein J and Pandolfi PP: Role of the proto-oncogene Pokemon in cellular transformation and ARF repression. Nature 433: 278-285, 2005.

13. Jeon BN, Yoo JY, Choi WI, Lee CE, Yoon HG and Hur MW: Proto-oncogene FBI-1 (Pokemon/ZBTB7A) represses transcription of the tumor suppressor $\mathrm{Rb}$ gene via binding competition with Sp1 and recruitment of co-repressors. J Biol Chem 283: 33199-33210, 2008.

14. Choi WI, Jeon BN, Park H, Yoo JY, Kim YS, Koh DI, Kim MH, Kim YR, Lee CE, Kim KS, et al: Proto-oncogene FBI-1 (Pokemon) and SREBP-1 synergistically activate transcription of fatty-acid synthase gene (FASN). J Biol Chem 283: 29341-29354, 2008.

15. Qu H, Qu D, Chen F, Zhang Z, Liu B and Liu H: ZBTB7 overexpression contributes to malignancy in breast cancer. Cancer Invest 28: 672-678, 2010.

16. Aggarwal H, Aggarwal A, Hunter WJ III, Yohannes P, Khan AU and Agrawal DK: Expression of leukemia/lymphoma related factor (LRF/Pokemon) in human benign prostate hyperplasia and prostate cancer. Exp Mol Pathol 90: 226-230, 2011.

17. Fang F, Yang L, Tao Y and Qin W: FBI-1 promotes cell proliferation and enhances resistance to chemotherapy of hepatocellular carcinoma in vitro and in vivo. Cancer 118: 134-146, 2012.

18. Jiao W, Liu F, Tang FZ, Lan J, Xiao RP, Chen XZ, Ye HL and Cai YL: Expression of proto-oncogene Pokemon in nasopharyngeal carcinoma cell lines and tissues. Asian Pac J Cancer Prev 14: 6315-6319, 2013.

19. Zhao GT, Yang LJ, Li XX, Cui HL and Guo R: Expression of the proto-oncogene Pokemon in colorectal cancer-inhibitory effects of an siRNA. Asian Pac J Cancer Prev 14: 4999-5005, 2013.

20. Liu F, Jiao W, Lan J and Xiao RP: Construction of short hairpin RNA recombinant plasmids targeting human Pokemon gene and screening in CNE2 cells. J Modern Oncol 22: 994-997, 2014 (In Chinese).

21. Liva KJ and Schmittgen TD: Analysis of relative gene expression data using real-time quantitative PCR and the 2(-Delta Delta C(T)) method. Methods 25: 402-408, 2001.

22. Song LB, Wang HM, Zeng MS, Li MZ and Jian SW: Study on the tumor heterogeneity of nasopharyngealcarcinoma cell line (SUNE-1). Ai Zheng 17: 324-326, 1998 (In Chinese).

23. Zhou W, Feng X, Ren C, Jiang X, Liu W, Huang W, Liu Z, Li Z, Zeng L, Wang L, et al: Over-expression of BCAT1, a c-Myc target gene, induces cell proliferation, migration and invasion in nasopharyngeal carcinoma. Mol Cancer 12: 53, 2013.

24. Deng YF, Zhou DN, Pan ZY and Yin P: Aberrant SATB1 expression is associated with Epstein-Barr virus infection, metastasis and survival in human nasopharyngeal cells and endemic nasopharyngeal carcinoma. Int J Clin ExP Pathol 7: 2454-2461, 2014.

25. Niu DL, Li JF, He F, Zou JT, Zhou QF, Wei X, Li Y and Chen LH: Influence of silencing Pokemon genes using small-interfering RNA on growth of CNE-2 cells of NPC. Chin J Cancer Prevention Treatment 9: 1041-1045, 2012 (In Chinese).

26. Ma LS, Yan QI, Huang Y, Zhao W and Zhu YU: Downregulation of human epidermal growth factor receptor 2 by short hairpin RNA increases chemosensitivity of human ovarian cancer cells. Oncol Lett 9: 2211-2217, 2015.

27. Tan X, He X, Jiang Z, Wang X, Ma L, Liu L, Wang X, Fan Z and Su D: Derlin-1 is overexpressed in human colon cancer and promotes cancer cell proliferation. Mol Cell Biochem 408: 205-213, 2015.

28. Chen CY, Lin YS, Chen CL, Chao PZ, Chiou JF, Kuo CC, Lee FP, Lin YF, Sung YH, Lin YT, et al: Targeting Annexin A2 reduces tumorigenesis and therapeutic resistance of nasopharyngeal carcinoma. Oncotarget 6: 26946-26959, 2015.

29. Talmadge JE, Wolman SR and Fidler IJ: Evidence for the clonal origin of spontaneous metastases. Science 217: 361-363, 1982.

30. Duda DG, Ancukiewicz M, Isakoff SJ, Krop IE and Jain RK: Seeds and soil: Unraveling the role of local tumor stroma in distant metastasis. J Natl Cancer Inst 106: pii: dju 187, 2014.

31. Xu T, Li ZM, Gu MF, Wei WH, Zhang GY, Wu QL, Su Y and Hu WH: Primary nasopharyngeal adenocarcinoma: A review. Asia Pac J Clin Oncol 8: 123-131, 2012.

32. Chen W, Lee Y, Wang H, Yu GG, Jiao W, Zhou W and Zeng Y: Suppression of human nasopharyngeal carcinoma cell growth in nude mice by the wild-type p53 gene. J Cancer Res Clin Oncol 119: 46-48, 1992. 
33. Teng ZP, Ooka T, Huang DP and Zeng Y: Detection of Epstein-Barr virus DNA in well and poorly differentiated nasopharyngeal carcinoma cell lines. Virus Genes 13: 53-60, 1996.

34. Xia Y, Wong NS, Fona WF and Tideman H: Upregulation of GADD153 expression in the apoptotic signaling of N-(4-hydroxyphenyl)retinamide (4HPR). Int J Cancer 102: 7-14, 2002.

35. Yang XL, Liu XC, Huang L, Lan J, Zhang HY, Qin MB, Zhong YY and Mo ZN: Effect of TSA on nasopharyngeal carcinoma CNE3 cells and its mechanism. Chin J Public Health 26: 1029-1030, 2010 (In Chinese).

36. Liu XC, Lan J, Nong CZ, Pan LL and Jiao W: Effect of mangiferin on induction of apoptosis and intracellular $\mathrm{Ca}^{2+}$ concentration in nasopharyngeal carcinoma CNE3 cells. Chin J New Clin Med 3 : 805-897, 2010 (In Chinese).

37. Peng LX, Chen JX, Cheng JJ, Liu F, Jiao W, Pang Q, Feng GS Li SG, Mo XY and Wu XX: Establishment of radioresistant subline from human nasopharyngeal carcinoma cell line by repeating irradiation. Chin J Clinical Med 5: 1107-1109, 2012 (In Chinese)
38. Liu XS, Haines JE, Mehanna EK, Genet MD, Ben-Sahra I, Asara JM, Manning BD and Yuan ZM: ZBTB7A acts as a tumor suppressor through the transcriptional repression of glycolysis. Genes Dev 28: 1917-1928, 2014.

39. Liu XS, Genet MD, Haines JE, Mehanna EK, Wu S, Chen HI, Chen Y, Qureshi AA, Han J, Chen X, et al: ZBTB7A suppresses melanoma metastasis by transcriptionally repressing MCAM. Mol Cancer Res 13: 1206-1217, 2015.

40. Zong D, Yin L, Zhong Q, Guo WJ, Xu JH, Jiang N, Lin ZR, Li MZ, Han P, Xu L, et al: ZNF488 enhances the invasion and tumorigenesis in nasopharyngeal carcinoma via the Wnt signaling pathway involving epithelial mesenchymal transition. Cancer Res Treat 48: 334-344, 2015. 